\title{
ASPECTOS DEL COMPORTAMIENTO DE FORRAJEO DE LA CIGÜITA DEL RÍO PARKESIA MOTACILLA (AVES: PASSERIFORMES: PARULIDAE) EN ÉPOCA NO REPRODUCTIVA
}

\author{
${ }^{1}$ Hodali Almonte-Espinosa y ${ }^{2}$ Steven C. Latta
}

\begin{abstract}
${ }^{1}$ Museo Nacional de Historia Natural de Santo Domingo (MNHNSD). Calle César Nicolás Penson, Plaza de la Cultura, Santo Domingo, República Dominicana. h.almonte@museohistorianatural.gov.do ${ }^{2}$ Department of Conservation and Field Research. The National Aviary. Allegheny Commons. Pittsburgh, PA, U.S.A. steve.latta@aviary.org
\end{abstract}

\section{RESUMEN}

Se estudió el comportamiento de forrajeo de Parkesia motacilla (Parulidae) durante los meses septiembre-noviembre y enero-marzo (2008-2009) en una localidad de la provincia Duarte, en el noreste de República Dominicana. Los aspectos considerados fueron: maniobras de alimentación, sustrato de forrajeo y tasa de forrajeo. Las maniobras de alimentación más utilizadas por la cigüita del río, en orden de frecuencia, fueron Picoteo Normal (PN), Picoteo Rápido (PR) y Picoteo al Aire (PA). En cuanto a los sustratos evaluados, encontramos que esta especie prefiere buscar sus alimentos sobre Rocas o Piedra (R-P) y Suelo o Arena (S). No se detectaron diferencias significativas en la frecuencia de forrajeo durante los períodos de estudio.

Palabras clave: Parkesia motacilla, maniobra de alimentación, sustrato de forrajeo, tasa de forrajeo, Río Bijao, Provincia Duarte, República Dominicana.

\section{ABSTRACT}

We studied the foraging behavior of Parkesia motacilla (Parulidae) during the months September to November and January to March in a village in the province Duarte in north-eastern, Dominican Republic. The aspects studied included: foraging maneuvers, foraging substrates, and foraging rate. The latter was estimated as the number of pecks given to the foraging substrate in five minutes. The foraging maneuvers most used were "normal picking" followed by "fast picking" and "air picking". Of the substrates evaluated, we found that this species prefers to seek their food on a rock or stone and soil or sand. We found no significant difference in the frequency of foraging during the periods of study.

Key words: Parkesia motacilla, foraging maneuver, foraging substrates, foraging rate, Bijao River, Duarte province, Dominican Republic.

\section{INTRODUCCIÓN}

En las aves, el forrajeo es una conducta trófica que incluye diferentes pautas: búsqueda, localización, persecución, captura, manipulación e ingestión de los alimentos. En tal sentido, su estudio involucra el conocimiento de los diferentes comportamientos alimentarios que caracterizan el aprovechamiento de recursos (Gutiérrez, 1998; Robinson y Holmes, 1982). El forrajeo en las aves está determinado por su anatomía, su morfología y las características del hábitat, así como la variación en la disponibilidad de alimentos (Robinson y Holmes, Ídem).

Por otra parte, elementos conductuales, como las maniobras que cada especie exhibe y los tipos de sustratos utilizados, son determinantes en el estudio del comportamiento de forrajeo (Remsen y Robinson, 1990; Colorado, 2004). Estos estudios son muy valiosos, ya que ayudan a explicar la destreza que tienen los individuos en la adquisición y uso de la energía de los 
recursos alimentarios (Hutto, 1990). Es importante un entendimiento de las diferencias entre especies e individuos en su comportamiento de forrajeo, ya que éste y las características del hábitat pueden indicar diferencias entre los tipos de recursos que las aves consumen (Robinson y Holmes, 1982). Además, la comprensión de estos aspectos podría ayudar a desarrollar mejores estrategias de conservación.

La cigüita del río (Parkesia motacilla) es un ave silvestre migratoria que sólo se encuentra en hábitats dulciacuícolas de alta calidad y de corrientes rápidas en las áreas de reproducción, por lo que ha sido utilizada como indicador de integridad biótica en estos ambientes (Mattsson y Cooper, 2006; Mulvihill et al., 2008). En los sitios de invernada suele encontrarse en vegetación ribereña cerca de arroyos y ríos de aguas claras y tranquilas en lugares montañosos y que, por lo general, presentan similitud con su área de cría (Master et al., 2005; Latta et al., 2006).

Los invertebrados acuáticos constituyen el principal recurso en la dieta de la cigüita del río, pero ésta también puede incluir insectos terrestres y otros invertebrados, como moluscos, e incluso se ha reportado que también se alimenta de anfibios (Craig, 1984; Latta et al., 2006; Gibson, 2007; Mulvihill et al., 2008).

Tanto en sus áreas de cría como de invernada, esta especie es considerada como un excelente indicador del buen estado de salud de hábitats, especialmente de las cabeceras de ríos (Mattsson y Cooper, 2006). Además, es una especie que ha sido declarada como especial para la conservación por el Servicio de Pesca y Vida Silvestre de los Estados Unidos (USFWS), debido a que sus poblaciones reproductoras están altamente localizadas por su extremada especificidad de hábitat.

Durante la estación reproductiva, se distribuye en el este de los Estados Unidos de Norteamérica, el sureste de la región de los grandes lagos (incluyendo sureste de Ontario y rara vez en el suroeste de Quebec) y el sureste de Nueva Inglaterra, así como a través de los estados del Golfo al norte de la Florida. En época no reproductiva migra desde el centro de México hacia el sur, hasta el centro de Panamá y el Caribe, también ha sido reportada para el noroeste de Colombia y norte de Venezuela (Latta et al., 2006; Gibson, 2007). En nuestra isla, ha sido reportada desde el nivel del mar hasta una altura máxima de 1,800 metros, pero prefiere arroyos de aguas frescas en montañas (Latta et al., 2006).

\section{OBJETIVOS}

- Evaluar la tasa de forrajeo en individuos de Parkesia motacilla durante los meses septiembre-noviembre y enero-marzo.

- Determinar si existen diferencias significativas en la tasa de forrajeo al principio de la temporada de invernada y al final de la misma.

- Determinar la frecuencia con que es utilizada cada maniobra de alimentación.

- Analizar preferencia por tipo de sustrato de forrajeo.

\section{MATERIALES Y MÉTODOS}

El trabajo de campo se realizó durante los meses de septiembre-noviembre del 2008 (primer período) y enero-marzo del 2009 (segundo período), en el río Bijao en Loma de la Joya, San Francisco de Macorís, Provincia Duarte, en el noreste de la República Dominicana. Hicimos observaciones focales directas con binoculares Celestron de Eagle 10x50 (10 de aumento y los objetivos de $50 \mathrm{~mm}$ de diámetro), una grabadora de mano modelo No: RP5032A de la marca RCA y un cronómetro modelo No: 75326, de la marca Q\&Q. El tiempo establecido para las observaciones fue desde las 07:00 hasta las 12:00 horas y desde las 15:00 hasta las 17:30 horas 
(la mayoría de las observaciones fueron realizadas en horas de la mañana). La distancia de observación fue de $5 \mathrm{~m}$ o más, con respecto al ave, para no interferir con su actividad. También se incluyeron observaciones oportunistas (fuera del tiempo de observación).

Los datos fueron tomados en intervalos de cinco minutos (denominados como eventos de observación), los cuales se contaron a partir del segundo intento de forrajeo (después que se observó el ave forrajeando por primera vez). Las referencias sobre los métodos y el número de actividad de forrajeo fueron hechas en base a Eaton (1958) y Craig (1984). En cada evento de observación se registraron las maniobras de alimentación, el sustrato de alimentación, la ubicación del alimento, el tipo de alimento, la distancia a partir de la orilla del río a la que el ave forrajea (en metros), el tiempo de forrajeo, el número de veces que el ave intenta comer algo y la distancia que el ave recorre mientras forrajea.

Para fines de este estudio, se hizo una modificación a las categorías de clasificación de los sustratos hecha por Craig (Ídem), quien los dividió en cuatro clases: agua (incluía alimentación en vegetación herbácea, madera seca y ramas), follaje, aire y suelo (incluía alimentación en rocas, hojarasca, barro y briófito). Nosotros dividimos los sustratos en nueve categorías, los detalles sobre las mismas se muestran en la Tabla1.

Tabla 1. Categoría de clasificación de sustratos.

\begin{tabular}{|ll|}
\hline Tipo de sustrato & Abreviatura \\
\hline Agua en movimiento & $\mathrm{MH}_{2} \mathrm{O}$ \\
Agua tranquila & $\mathrm{MNH}_{2} \mathrm{O}$ \\
Roca o Piedra & $\mathrm{R}-\mathrm{P}$ \\
Roca cubierta con vegetación & $\mathrm{RV}$ \\
Rama seca & $\mathrm{RS}$ \\
Encima de hojas & $\mathrm{EH}$ \\
Suelo o Arena & $\mathrm{S}$ \\
Árbol & $\mathrm{A}$ \\
Aire & $\mathrm{AR}$ \\
\hline
\end{tabular}

El sustrato A incluía tallos, hojas y vegetación herbácea, RS contenía pedazos de madera, troncos secos y partes secas de árboles vivos, $\mathrm{S}$ se refiere al suelo o la arena a la orilla del río, EH al suelo o arena cubierto con hojarasca, así como hojas en la orilla y RV incluye rocas cubiertas de briófitos u otro tipo de vegetación. Dichas rocas se encontraban dentro del río en la mayoría de los casos. El sustrato $\mathrm{MH}_{2} \mathrm{O}$ incluyó el agua en movimiento y hojas dentro del agua, mientras que el sustrato $\mathrm{MNH}_{2} \mathrm{O}$ incluyó aguas sin movimiento aparente y hojas dentro del agua, mientras que R-P solo incluyó rocas o piedras.

En base a sus observaciones, Craig (1984) evaluó cuatro maniobras de forrajeo: picoteos o recolección, tirones de hoja, halconeo y balanceo. Para fines de esta investigación, dicha clasificación fue modificada, los detalles sobre la clasificación de las maniobras evaluadas se muestran en la Tabla 2. 
Tabla 2. Categorías de clasificación para las maniobras de forrajeo.

\begin{tabular}{|c|c|c|}
\hline Maniobras de alimentación & Abreviatura & Descripción \\
\hline Picoteo normal & $P N$ & Los picoteos pueden contarse \\
\hline Picoteo rápido & $P R$ & Los picoteos no pueden contarse, porque no se ven \\
\hline Picoteo al aire & $P A$ & $\begin{array}{l}\text { El ave picotea al aire mientras está posada en otro } \\
\text { sustrato }\end{array}$ \\
\hline Saliendo al aire & $S A$ & El ave vuela para capturar alimento en el aire \\
\hline $\begin{array}{l}\text { Saliendo de un } \\
\text { sustrato a otro }\end{array}$ & $S P$ & $\begin{array}{l}\text { El ave vuela para capturar alimento, pero de un } \\
\text { sustrato diferente del aire }\end{array}$ \\
\hline Corre y captura & $C C$ & $\begin{array}{l}\text { El ave persigue una presa; el sustrato de } \\
\text { alimentación puede ser el aire o el suelo }\end{array}$ \\
\hline
\end{tabular}

La frecuencia de cada maniobra de forrajeo se determinó dividiendo el número de intentos con esa maniobra entre el total de intentos observados durante la investigación, incluidas todas las maniobras (Rodríguez-Camacho, 2006). La tasa de forrajeo fue determinada utilizando el tiempo de observación entre el número total de intentos de forrajeo registrado (Latta y Wunderle, 1996; Wunderle y Latta, 1998). Comparamos la tasa de forrajeo registrada durante los meses septiembre-noviembre y enero-marzo (2008-2009) por medio de una prueba T de Student para muestras independientes. Realizamos este análisis con el paquete estadístico Statistical Package for the Social Sciences (SPSS).

\section{RESULTADOS}

Durante 92 horas de observación, registramos 61 intentos de alimentación en un total de 13 individuos. Encontramos que PN fue la maniobra de alimentación más utilizada con un 58.97\% en el primer período y $52.94 \%$ en el segundo período del total registrado, seguida de PR y PA, la primera con $15 \%$ en ambos períodos y la segunda con $10 \%$ y $32 \%$ respectivamente. Los valores restantes corresponden a las demás maniobras, las cuales sólo fueron registradas durante septiembre-noviembre.

Las maniobras PN y PR nunca fueron registradas para el sustrato AR. Las PA y SP, como era de suponerse, solo fueron registradas para el sustrato AR, la maniobra CC fue registrada para el aire y suelo. De los métodos de forrajeo, PN, PR y PA fueron los más utilizados por Parkesia motacilla.

R-P resultó ser el sustrato al cual la cigüita del río dirigió con mayor frecuencia sus maniobras de alimentación. Le siguieron a éste, en orden de importancia, $\mathrm{EH}, \mathrm{S}, \mathrm{MH}_{2} \mathrm{O}, \mathrm{RV}, \mathrm{A}$ y $\mathrm{MNH}_{2} \mathrm{O}$. El porcentaje registrado para cada uno de los sustratos evaluados se muestra en detalle en la figura 2 . 
Septiembre-noviembre

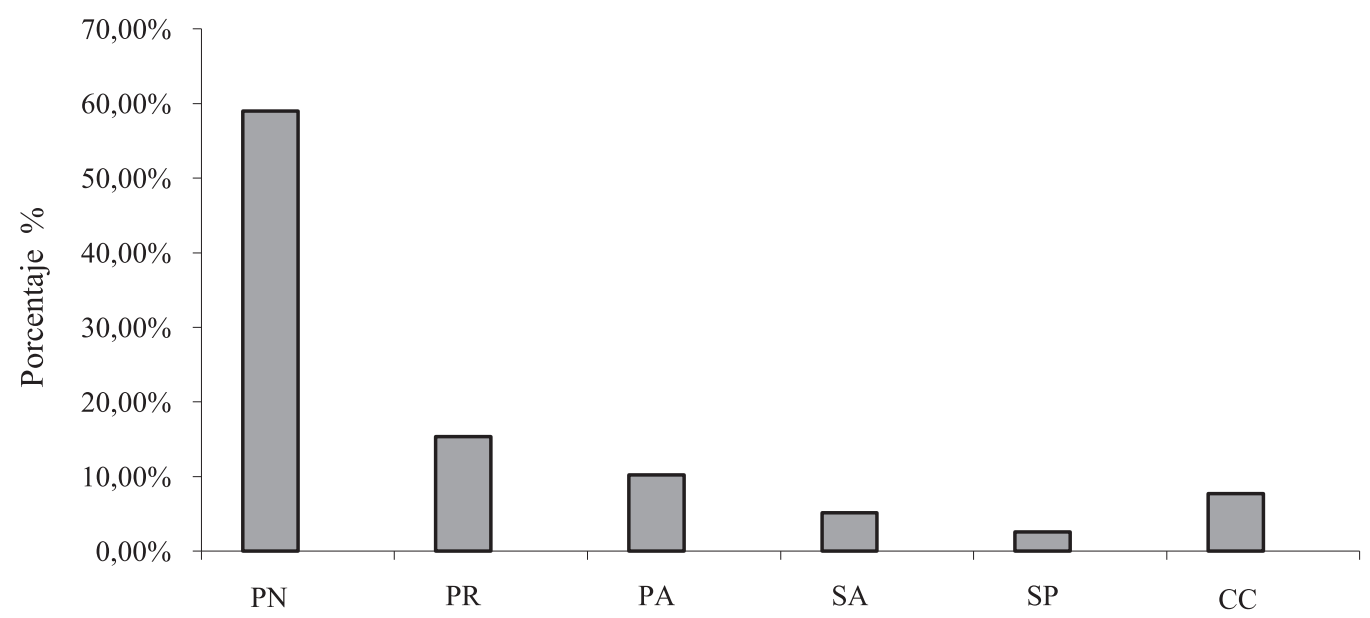

Maniobras de forrajeo

Enero-marzo

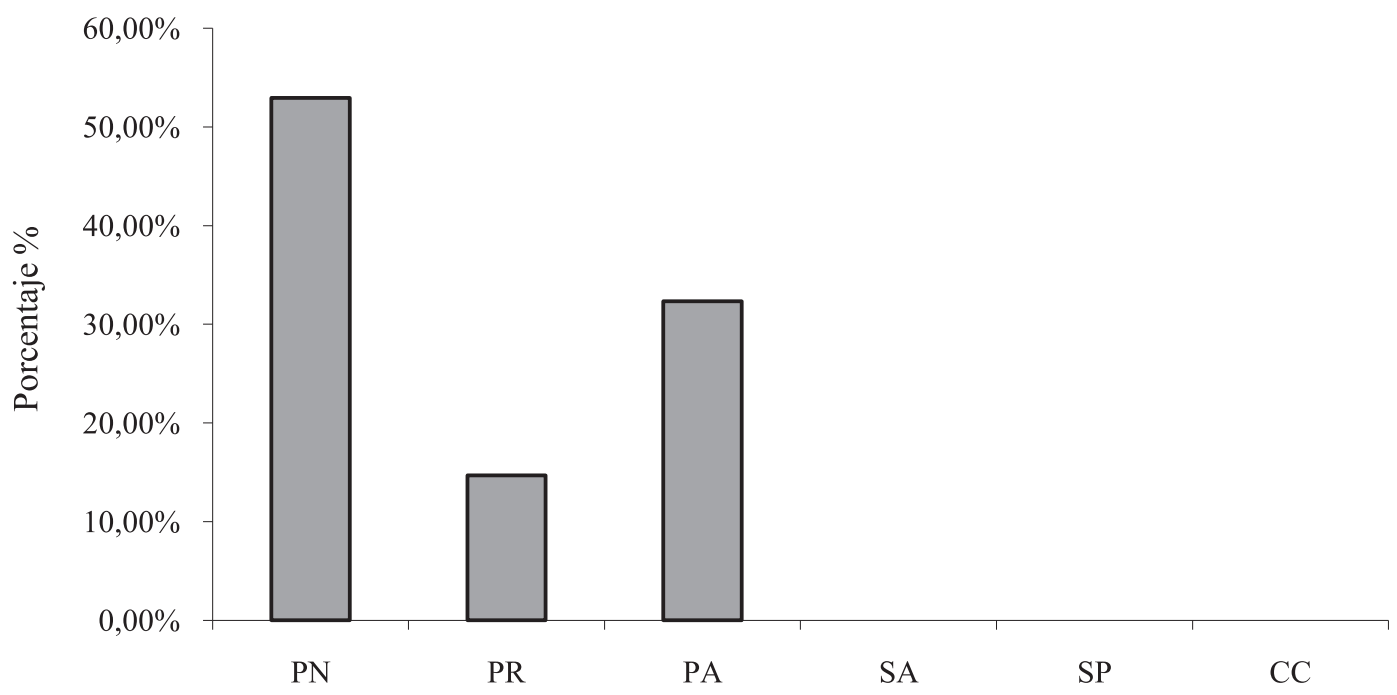

Maniobras de forrajeo

Figura 1. Frecuencia de uso de maniobras de forrajeo, en base a los datos obtenidos durante el período 2008-2009.

Encontramos que esta especie, mientras forrajea, se mueve a una distancia promedio de $1.4 \mathrm{~m}$ buscando comida y que puede recolectar alimento a una distancia de $2.6 \mathrm{~m}$ de la orilla del río. De los 61 intentos de alimentación observados, solo en seis ocasiones pudimos identificar el alimento capturado, en la mayoría de los casos fueron invertebrados (lepidópteros, dípteros y anélidos) atrapados en maderas secas, tronco de árboles, aire, árboles, suelo y rocas. 
Septiembre-noviembre

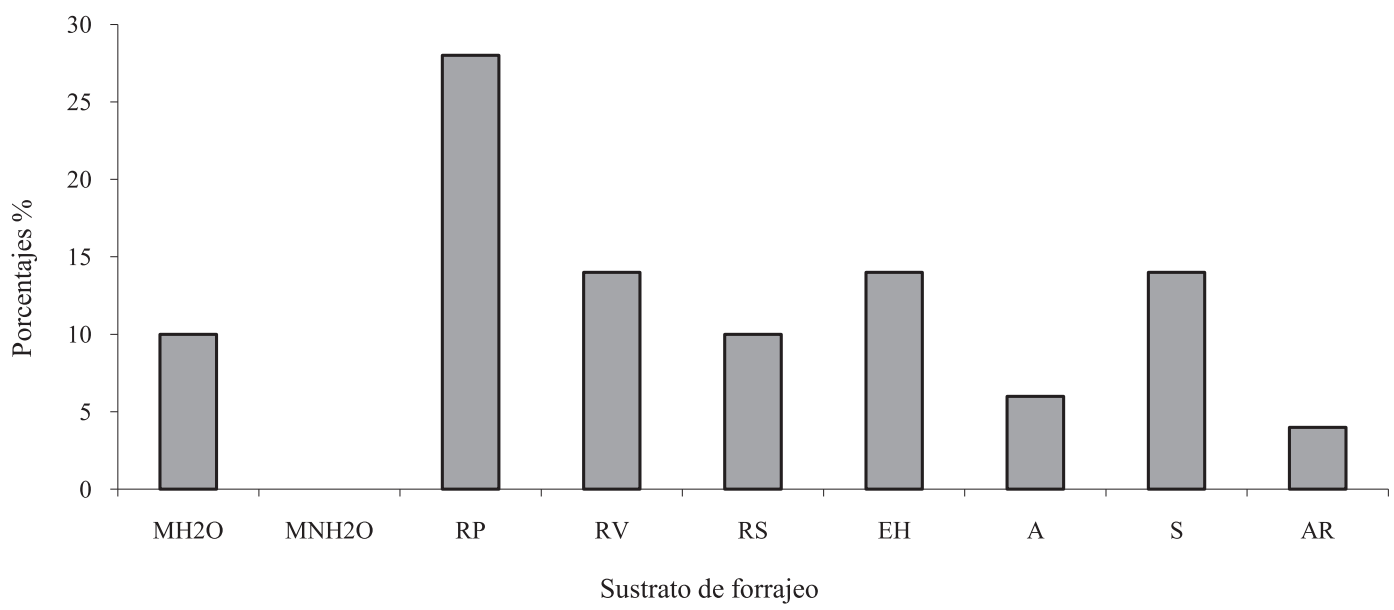

Enero-marzo

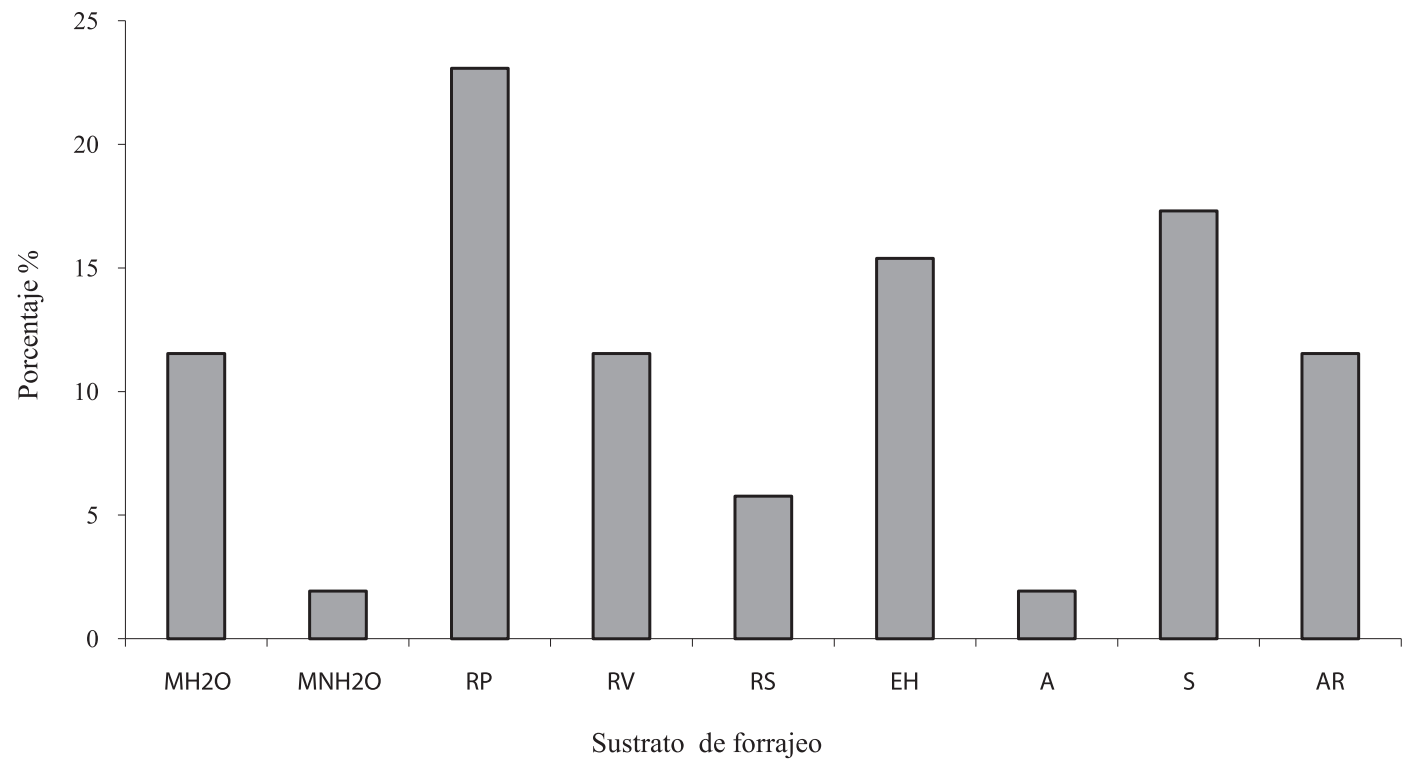

Figura 2. Uso de sustrato de forrajeo, en base a los datos obtenidos en 2008-2009.

Tabla 3. Detalles sobre observaciones focales de Parkesia motacilla en Río Bijao, Provincia Duarte, República Dominicana.

\begin{tabular}{|llll|}
\hline Período de estudio & $\mathrm{N}^{*}$ & $\begin{array}{l}\text { Promedio de } \\
\text { picotazos }\end{array}$ & $\begin{array}{l}\text { Tiempo promedio de forrajeo } \\
\text { (minutos y segundos) }\end{array}$ \\
\hline Septiembre-noviembre & 13 & 14.19 & $4: 30$ \\
Enero-marzo & 12 & 10.56 & $2: 42$ \\
\hline
\end{tabular}

$* \mathrm{~N}=$ número de individuos observados. 
No se registraron diferencias significativas en la tasa de forrajeo para $P$. motacilla durante los períodos septiembre-noviembre 2008 y enero-marzo $2009(T=1.307, d f=60, P=0.196)$.

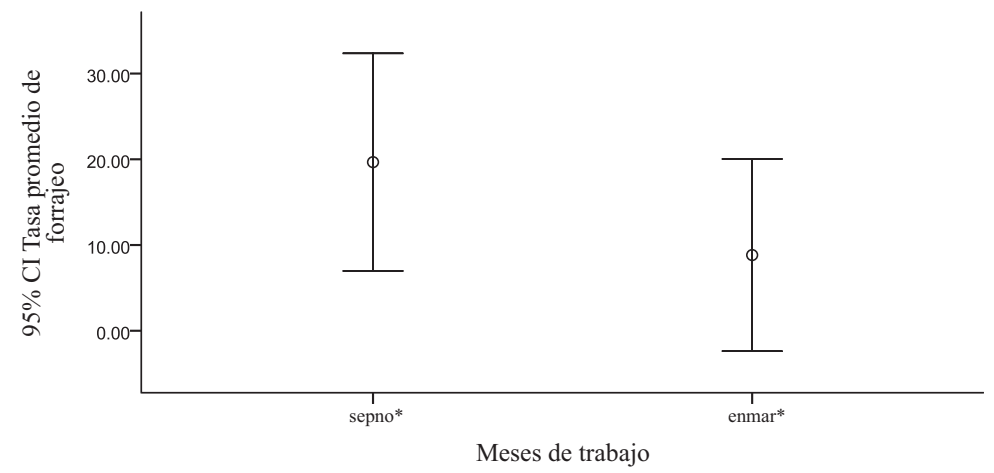

Figura 3. Frecuencia de forrajeo durante los períodos de estudio.

*sepno: septiembre-noviembre, enmar: enero-marzo.

\section{DISCUSIÓN Y CONCLUSIONES}

El análisis sobre la preferencia alimentaria de la cigüita del río nos muestra que esta especie utiliza con más frecuencia la maniobra de forrajeo Picoteo Normal (PN), seguida de Picoteo Rápido (PR) y Picoteo al Aire (PA). Estos resultados concuerdan con los obtenidos por Craig (1984), quien encontró que la cigüita del río (Parkesia motacilla) depende casi exclusivamente de la maniobra de alimentación Picoteo; este autor incluía dentro de la maniobra Picoteo la de Picoteo Rápido (PR), evaluada por nosotros de manera independiente. Craig encontró que la maniobra Tirones de Hojas se presentaba en segundo lugar de frecuencia, esta maniobra de forrajeo nosotros la incluimos como parte de Picoteo Normal (PN).

El tipo de sustrato más utilizado por la cigüita del río, de acuerdo a nuestro trabajo, es el de Roca o Piedra (R-P), difiriendo este resulatado del obtenido por Craig (Ídem), quien registra el sustrato Agua como el más utilizado. La posible explicación a esta diferencia se debe a que Craig incluyó la orilla del río dentro del sustrato agua, mientras que para nosotros es parte del sustrato Suelo o Arena (S); Craig, a su vez, incluye en este mismo sustrato vegetación herbácea (para nosotros parte de A, Árbol) y madera seca (para nosotros RS), así como ramas (parte de A), mientras que nosotros lo evaluamos como sustratos independientes o estaba dentro de otra categoría.

La identificación del alimento al momento de las observaciones nos muestra que esta especie prefiere alimentarse de invertebrados. Esto coincide con lo encontrado por Craig (1984); Latta et al. (2006) y Gibson (2007), quienes presentan a los invertebrados acuáticos y terrestres como la principal fuente de alimento de Parkesia motacilla.

En cuanto a la distancia a la que el ave forrajea de la orilla del río, nuestros resultados concuerdan con lo encontrado por Eaton (1958) y Latta et al. (2006), quienes encontraron que esta especie prefiere las periferias de arroyos y lagos de aguas frescas, limpias y rápidas en montañas.

Por otra parte, a pesar de la similitud en la tasa de alimentación, durante el período septiembrenoviembre, los individuos de esta especie dedicaron más tiempo al forrajeo, probablemente porque necesitaban reponer la energía invertida durante su viaje hacia sus áreas de invernada. 
Además, la similitud en la tasa de forrajeo puede estar relacionada con la necesidad de reponer la grasa consumida durante la migración hacia sus áreas de invernada y la obtención de la misma para el viaje hacía sus áreas de reproducción. Otra posibilidad es que no se hayan detectado diferencias significativas debido a que las aves probablemente aumentan su tasa de obtención de alimento días antes de la migración, lo que no quedaría registrado al no incluir esta investigación el mes de marzo completo; aunque se desconoce la posible fecha de retorno de esta especie a sus áreas de reproducción, pero se supone que regresan a finales de marzo o principios de abril.

Conocer las preferencias alimentarias de la cigüita del río y sus estrategias de alimentación, así como el hecho de que ésta prefiere alimentarse sobre rocas, suelo y entre hojas en la periferia de ríos y arroyos, es un buen punto para el desarrollo de planes de conservación de esta peculiar especie y de sus hábitats.

\section{AGRADECIMIENTOS}

Este estudio fue posible gracias al apoyo del Aviario Nacional de los Estados Unidos de Norteamérica y el Museo Nacional de Historia Natural de Santo Domingo. Danilo Mejía, María Paulino, Eudy Paulino y Ángel Paulino contribuyeron significativamente en la realización del trabajo. Carlos Suriel, Solanlly Carrero y Miguel Santiago Núñez hicieron comentarios al manuscrito. A todos ellos nuestros más sinceros agradecimientos.

\section{LITERATURA CITADA}

Colorado, G. 2004. Relación de la morfometría de las aves con gremios alimenticios. 25 Boletín SAO. 26 (27): 25-32.

Craig, R. 1984. Comparative foraging ecology of Louisiana and Northern waterthrushes. Wilson Bull 1 Vol. 96, No. 2, June 1984.

Eaton, S . W. 1958. A life history study of the Louisiana Waterthrush. Wilson Bull. 70: 211-236.

Gibson, J. 2007. Special animal abstract for Louisiana Waterthrush. (Seiurus motacilla) Michigan Natural Features Inventory, Lansing, MI.

Gutiérrez, G. 1998. Estrategia de forrajeo. Manual de análisis experimental del comportamiento. 359-381 pp.(http://www.docentes.unal.edu.co/gagutierrezd/docs/Publicaciones GGutierrez/1998\%20Estrategias\%20de\%20Forrajeo.pdf). Fecha de consulta: 26-V-2009.

Hutto, R. L. 1990. Studies of foraging behavior: central to understand the ecological consequences of variation in food abundance. Studies in Avian Biology 13: 389-390.

Latta, S. C., and J. M. Wunderle, Jr. 1996. The composition and foraging ecology of mixedspecies flocks in pine forests of Hispaniola. Condor 98: 595-607

Latta, S., C. Rimmer, A. Keth, J. Wiley, H. Raffaele, K. Mcfarland y E. Fernández. 2006. Aves de la República Dominicana y Haití. Princeton University Press, Vii+258 pp.

Master, T., R. Mulvihill, L. Robert, J. Sanchez y E. Carman. 2005. A Preliminary Study of Riparian Songbirds in Costa Rica, with Emphasis on Wintering Louisiana Waterthrushes. USDA Forest Service Gen. Tech. Rep. PSW-GTR-191 (1): 528-531. 
Mattsson, B. y R. Cooper J. 2006. Louisiana waterthrushes (Seiurus motacilla) and Habitat Assessments as Cost-effective Indicators of Instream Biotic Integrity. Freshwater Biology 51 (10): 1941-1958.

Mulvihill, R. S., F. L. Newell, and S. C. Latta. 2008. Effects of acidification on the breeding ecology of a stream-dependent songbird, the Louisiana Waterthrush (Seiurus motacilla). Freshwater Biology, 53: 2158-2169

Remsem, J. y S. Robinson. 1990. A Classification Scheme for foraging Behaviour of Birds in Terrestrial Habitats. Studies in Avian Biology, (13): 144-160.

Robinson, S. K. y R. T. Holmes. 1982. Foraging behavior of forest birds: the relationships among search tactics, diet, and habitat structure. Ecology, 63: 1918-1931.

Rodríguez-Camacho, E. 2006. Ecología del Todus mexicanus (Coraciiformes-Todidae) en el Bosque de Susúa, Sabana Grande, Puerto Rico. Tesis de maestría Universidad de Puerto Rico Recinto Universitario de Mayagüez.

Wunderle, J. M., Jr. y S. C. Latta. 1998. Avian resource use in Dominican shade coffee plantations. Wilson Bulletin, 110: 255-265 